\title{
Enhancing User Immersion and Virtual Presence in Interactive Multiuser Virtual Environments through the Development and Integration of a Gesture-Centric Natural User Interface Developed from Existing Virtual Reality Technologies
}

\author{
Chika Emma-Ogbangwo, Nick Cope, Reinhold Behringer, and Marc Fabri \\ School of Computing Creative Technology and Engineering \\ Faculty of Arts, Environment and Technology \\ Leeds Metropolitan University, \\ Leeds, United Kingdom \\ c.emma-ogbangwo2888@student. leedsmet.ac.uk, \\ \{n.cope, r.behringer,M.Fabri\}@leedsmet.ac.uk
}

\begin{abstract}
Immersion, referring to the level of physical or psychological submergence of a user within a virtual space relative to that user's consciousness of the real-world environment, has predominantly been established as an indispensable part of interactive media designs. This is most prevalent in Virtual Reality (VR) platforms, as their applications are typically reliant on user believability. With a wide variation of possible methodologies for the enhancement of this feature, the collectively recognised paradigm lies on the emphasis of naturalism in the design of the virtual system [7].

Though widely used by some specialised VR applications [4] such concepts are yet to be fully explored in the more contemporary virtual systems such as Social Immersive Virtual Environment (SIVE).

The focus of the study described in this paper are the techniques being developed to enhance user immersion, virtual presence and co-presence in a SIVE application, through the design and integration of a VR-based Natural User Interface (NUI) that allows users to naturally and intuitively interact with the virtual environment and other networked users through the utilisation of full body gesture controls. These gestural controls prioritise the emulation of the alternate equivalent of such real-wold interactions, whilst also providing an interface for the seamless and unobtrusive translation of the user's real-world physical state into the virtual environment through intuitive user to virtual avatar proprioceptive coordination.
\end{abstract}

\section{$1 \quad$ Introduction}

With the increasing advancement in digital computing technologies comes an equivalent increase in the processing resources available to Virtual Environment (VE) developers, consequently resulting in its increase in complexity. For SIVE 
applications, however, there is a need for the inclusion of additional subsystem that effectively translates the user's real-world physical state upon their actualised virtual representation, consequently leading to the introduction of a Natural User Interface (NUI) paradigm for the sustainability of user immersion and virtual presence. While there have been a significant number of studies utilising the NUI design concept [4], an invariably high number of such studies are typically focused on its integration within the more specialised VR applications, with virtual systems such as the SIVE platform still utilising conventional 2D UIs. Notable limitations of such 2D UIs are in the form of a disconnection between performed user actions and its resulting effects on the reactive VE. In SIVE applications, these limitations are significantly more prevalent as certain UI functionalities, particularly those required for inter-user communication, cannot be effectively adapted to such UI due to the obvious limitation of their implemented technologies.

Mitigating such limitations is the primary focus of this study, as it seeks to utilise the NUI design concepts within a SIVE application, intuitively adapting its immersive VR technologies for the development and integration of an NUI architecture that allows for seamless and unobtrusive user virtual interaction, perception, embodiment and inter-user communication, within a perceptively shared immersive virtual space. Its approach primarily seeks to focus on the emulation of natural real-world interaction and communication paradigms within its SIVE's Interaction, Perception, and Embodiment UIs.

\section{Related Works}

While the integration of the NUI design concept in VR and other immersive applications can be considered as being relatively new, a significant number of studies have been done in this area with their respective scope ranging from the focus on a single component within the UI to the development of multimodal architectures. Among these studies include HandNavigator device [10] and Piivert device [3], with [10] focusing on kinesthetic virtual interaction, [3] combining the Ray-Casting technique with a finger gesture UI. The implementation of the hybrid UI concepts is a relatively common methodology in immersive VR development, as illustrated by EyeMotion interface [8], which integrated gaze tracking technology with a gesture recognition and mouse input interface.

With regards to user virtual navigation, methodologies for UI integration can generally be classified as either Position Tracking Interfaces, or User Controlled Interfaces. Notable work done on position tracking in immersive VR include Redirection Technique [12], which constituted the dynamic adjustment of the user's translational and rotational movements within the VE in order to compensate for the limited available real-world space. Other methodologies from related studies include Immersive Navidget [9], and the Step WIM interface [11]. Though typically not prioritised by most NUI developers, there has, however, been some notable work done on the immersive embodiment UI, with some methodologies developed by previous studies including haptic user kinesthetic interface [1], Puppet UI concept [6], and proprioceptive embodiment UI [5], which utilised the Vicon motion tracking system. 


\section{$3 \quad$ Research Methodology}

Considering the limitations of the methodologies employed in the current state of the art, this study's approach to the integration of the NUI concept within a VR SIVE application (developed via Crytek's CryEngine 3 SDK) focuses on emphasising the emulation of natural real-world interaction and communication paradigm within the $\mathrm{VE}$, through an unobtrusive gesture-centric user control interface.

\subsection{The Interaction UI}

Gesture sequences configured in the Interaction UI utilise a combination of single and two-handed virtual interaction technique, while position translational gestures implements a series of static user leg gestures that natural depict the intended direction of movement. For user perspective viewpoint control, however, the interaction UI implements a hybrid architecture, consisting of a hand motion tracking and head tracking system respectively. Through the Kinect device, the hand tracking system incorporates a series of static hand gestures that naturally depict the intended direction of viewpoint orientation, while the alternate head tracking system makes use of NaturalPoint Inc's TrackIR 5 (6DOF) and Trackclip Pro head tracking device, for viewpoint orientation control.

\subsection{The Embodiment UI}

Utilised for the visualisation of avatar Non-Verbal Communication (NVC) features within the SIVE, the embodiment UI comprises of the Autonomous Non-Verbal Communication (ANVC) system, the Motor Spatial Mapping (MSM) system, and the avatar Lip-Sync Emulation (LSE) system. Consisting of a series of static (MSM) and dynamic (ANVC) gesture libraries, synchronously tracked and recognised, the MSM and ANVC systems allowed for natural user to avatar motor channel proprioceptive coordination, and the LSE system used for the visualisation of avatar lip-syncing functionalities.

\subsection{Designing the GMA API}

Written in C\# programming language, and developed via Microsoft Visual Studio 2012's .NET Framework, the GMA API is a custom designed software application created and implemented for the purpose of interfacing the NUI's immersive VR technology with a target SIVE platform. Interfaced with the NUI's VR technology and the SDK's SIVE application through Open Sound Control (OSC) networking, the GMA is utilised in the integration of the interaction UI's gesture-based VE interaction and navigation interface, the embodiment UI's ANVC, MSM, and LSE sub-systems, and the perception UI's user crossConnection system. In order to achieved precise gesture recognition the GMA consist of three independently developed interrelated APIs comprising of the GMASynthesiser, the GMARecognizer.dll class libraries, and the GesturePak API. 


\subsection{Research Testing and Evaluation}

While this study is currently part of an ongoing research, its intended methodology for evaluation would require three distinct VR setups, including the NUI HMD-Based $V R$ (for Discrete Evaluation data), the NUI Desktop-Based VR (used for Experiment Control data), and the Desktop VR (for analysis Baseline data). Testing procedures would involve the engagement of participants through multiple Virtual Scenarios $(V S)$, consisting of VS1, VS2, and VS3, incorporated for the evaluation of immersion, interface usability, virtual presence and co-presence, through a communicative, natural interactive and contemporary IVE virtual scenarios respectively. Adopting the triangulation methodology, quantitative data being measured would be the participants' Heart Rate Variation (HRV) values, critically evaluated via a proposed Response Expectancy (RE) concept, which would seek to correlate expected changes in participants' HRV values to its actual changes relative to the current state of the SIVE. Supporting qualitative analysis would utilise Presence Evaluation and Immersive Tendency Questionnaires and interviews.

\section{Conclusion}

This study presents a gesture-centric NUI architecture that aims at emphasising natural virtual interaction and inter-user communication within a SIVE application, for the purpose of enhancing its user immersive quality. To achieve this, the GMA API was developed and utilised in the integration of a multi-tiered UI architecture consisting of a multimodal Interaction UI, a proprioceptive Embodiment UI and an immersive Perception UI. While the study is currently part of an ongoing research, critical evaluation of the developed NUI architecture would adopt a triangulation methodology, implementing quantitative measures via a proposed $R E H R V$ concept, and qualitative measures done through suitable $I T Q$ and $P Q$ templates.

\section{References}

1. Ahn, S.J.: Embodied Experiences in Immersive Virtual Environments: Effects on ProEnvironmental Attitude and Behaviour (2011), http://vhil.stanford.edu/ pubs / 2011 /ahn-embodied-experiences . pdf (accessed March 13, 2014)

2. Banos, R.M., et al.: Immersion and Emotion: Their Impact on the Sense of Presence (2004), http: / / www. itu.dk/ khhp/Konceptudvikling/Banos , 20 R\% $20 \mathrm{M}, \% 20 \mathrm{mfl} \div 20-$

$\% 20$ Immersion $\% 20$ and $\% 20$ Emotion $\% 20$ Their $\% 20$ Impact $\% 20$ on 20 the 20 . pdf (accessed March 17, 2014)

3. Berthaut, F., Hachet, M., Desainte-Catherine, M.: Piivert: Percussion-based Interaction for Immersive Virtual Environments (2010), http://www.researchgate.net/ profile/Martin_Hachet/publication/224129938_Piivert_Percussi on-based_interaction_for_immersive_virtual_environments / file/504635156a0986c566.pdf (accessed March 12, 2014) 
4. Deller, M., Ebert, A., Bender, M., Hagen, H.: Flexible Gesture Recognition for Immersive Virtual Environments (2006), http://www.researchgate.net/publication/ 4247845_Flexible_Gesture_Recognition_for_Immersive_Virtual_E nvironments/file/72e7e51a5f0769ba3b.pdf (accessed February 03, 2014)

5. Dodds, T.J., Mohler, B.J., Bulthoff, H.H.: Talk to the Virtual Hands: Self-Animated Avatars Improve Communication in Head-Mounted Display Virtual Environments) (2011), http://www.plosone.org/article/fetchobjectAttachment. action?uri=info\%3Adoi 2 F10.1371\%2Fjournal . pone. $0025759 \& r e p r e$ sentation=PDF (accessed March 13, 2014)

6. Eirik, F., Xin, L.: The Puppet UI: Tools for Nonverbal Communication in Virtual Environments (2007), http://www. thoughtbird.com/portfolio/puppetui /puppetui_paper.pdf (accessed March 13, 2014)

7. Hillaire, S., Lecuyer, A., Cozot, R., Casiez, G.: Using an Eye-Tracking System to Improve Camera Motions and Depth-of-Field Blur Effects in Virtual Environments (2008), http://people.rennes.inria.fr/Anatole.Lecuyer/vr08_hillaire. pdf (accessed February 03 2014)

8. Jimenez, J., Gutierrez, D., Latorre, P.: Gaze-based Interaction for Virtual Environments (2008), http://giga.cps.unizar.es/ diegog/ficheros/pdf_papers/ final.pdf (accessed March 12, 2014)

9. Knodel, S., Hachet, M., Guitton, P.: Navidget for Immersive Virtual Environments (2008), http: / / iparla.inria.fr/publications/2008/KHG08a/paper.pdf (accessed March 13, 2014)

10. Kry, P.G., et al.: HandNavigator: Hands-on Interaction for Desktop Virtual Reality (2008), http://www.cs.mcgill.ca/ kry/pubs/hn/hn.pdf (accessed March 12, 2014)

11. LaViola, J.J., et al.: Hands-Free Multi-Scale Navigation in Virtual Environments (2001), http://cs.brown.edu/ jjl/pubs/i3d01_laviola.pdf (accessed March 13, 2014)

12. Suma, E.A., et al.: A Taxonomy for Deploying Redirection Techniques in Immersive Virtual Environments (2012), http://ict.usc.edu/pubs/A\%20Taxonomy $\% 20$ for $\% 20$ Deploying $\% 20$ Redirection\% 20 Techniques $\% 20$ in $\% 20$ Immersi ve\%20Virtual\%20Environments.pdf (accessed March 12, 2014) 